\title{
Insect Sting Allergy
}

\author{
Alfredo Arias-Cruz* \\ Regional Center of Allergy and Clinical Immunology, "Dr. José Eleuterio González" University Hospital, Autonomous University of Nuevo León, \\ Monterrey, Nuevo León, Mexico
}

Most of the reactions caused by insect stings are usually local reactions of limited extension, tolerable, and self-limited. However, it is estimated that $15 \%$ or more of the people who suffer insect stings may present large local reactions, which are characterized by being $>10 \mathrm{~cm}$ in diameter, surrounding the location of the sting, and lasting more than $24 \mathrm{~h}$. On the other hand, insect stings may also be a cause of systemic reactions, which in some cases can be lethal ${ }^{1,2}$.

Even though there is a wide variety of insects whose sting may cause allergic reactions, the ones who most frequently cause this belong to the Hymenoptera order, which includes bees, bumble bees, wasps, hornets, and ants. The frequency of allergic reactions caused by insect stings from different families of Hymenoptera varies depending on the world region'.

The prevalence of systemic reactions caused by Hymenoptera stings varies from $0.4 \%$ to $0.8 \%$ in children and from $3 \%$ to $4 \%$ in adults. These reactions represent up to $34.1 \%$ of all causes of anaphylaxis. Approximately $20 \%$ of deaths by anaphylaxis are a result of a Hymenoptera sting. Estimations suggest that, as a result, there are at least 40 deaths per year in the US alone, while in Europe, only between 0.03 and 0.48 deaths per million inhabitants are caused by this ${ }^{1,3,4}$.

Systemic reactions may be mild when they are limited to cutaneous symptoms (itching, erythema, urticaria, and angioedema), and severe when there is respiratory (throat or laryngeal edema and bronchospasm), or cardiovascular (hypertension, shock, and arrhythmias) affectation. Some patients may also present gastrointestinal (nausea, vomiting, diarrhea, and abdominal pain) or neurological (seizures) manifestations ${ }^{1,3}$.

The factors, which influence the severity of the allergic reaction to hymenopterous venom, include a previous history of severe systemic reactions, type of insect (greater with bee stings), advanced age, preexisting respiratory or cardiovascular diseases, systemic mastocytosis, and the use of some medications; like beta-blockers or angiotensin-converting enzyme inhibitors. On the contrary, patients with a history of large local reactions or mild systemic reactions (especially children), as well as those who receive specific venom immunotherapy, have a lower risk of presenting severe allergic reactions ${ }^{1,3-6}$.

Diagnosis of allergy to Hymenoptera venom is based on clinical history and the identification of sensitization through skin tests or specific serum IgE determination. This diagnostic approach is enough for most patients to decide on a treatment. However, there are cases of anaphylaxis with negative specific $\lg E$ and those where there are multiple sensitizations, posing a unique diagnostic challenge. The future use of recombinant allergens could improve the diagnosis and facilitate the identification of specific sensitization in cases where there is cross-reactivity between venoms of different families, genus, or species of Hymenoptera. Tests to detect the activation of basophils could also be useful in the diagnostic evaluation of patients with a history of systemic reactions caused by Hymenoptera stings.

Specific venom immunotherapy, or with whole-body extract in the case of ants, is currently the only

\section{Correspondence:}

Alfredo Arias-Cruz

E-mail: aarias45@hotmail.com
Available online: 01-10-2018

Date of reception: 02-03-2018

Date of acceptance: 20-03-2018

DOI: 10.24875/RMU.M19000031
Medicina Universitaria. 2018;20(2):99-100 www.medicinauniversitaria.org CC BY-NC-ND license (http://creativecommons.org/licenses/by-nc-nd/4.0/). 
treatment that may prevent morbidity and mortality, maintains a low risk of systemic reaction during and after treatment, and improves quality of life. The protection derived from venom immunotherapy is effective once the maintenance dose is reached. Factors which may condition incomplete protection include allergies to bee venom, an insufficient dose of the venom (thus, in some cases, it is necessary to increase the dosage from $100 \mathrm{mc} \mathrm{g}$ to $200 \mathrm{mc} \mathrm{g}$ ), elevated basal serum tryptase, the coexistence of mastocytosis, and a repeated occurrence of adverse reactions during immunotherapy ${ }^{1,6}$.

Venom immunotherapy is indicated in those cases where there is a history of severe systemic reaction and sensitization is proven through skin tests or positive specific serum IgE determination for the venom of the insect implicated in the reaction. Indication of immunotherapy in mild systemic reaction cases will depend on the existence of risk factors (concomitant diseases or medications) or anxiety, which significantly affects the patients' quality of life. Nevertheless, in general, specific immunotherapy is not recommended in cases with large local reactions; it could be considered in patients with a greater risk of exposure to the implicated Hymenoptera stings, or those cases where the patients' quality of life may possibly be significantly affected. Some studies suggest the possible use of sublingual immunotherapy in these cases ${ }^{1,3,6}$.

Long-term follow-up studies have shown that in most patients, protection lasts for several years after having received immunotherapy with venom (between 3 and 5 years). Patients with a higher risk of recurrence of systemic reactions despite having received immunotherapy are those with a previous history of severe reaction before and during treatment (either by immunotherapy itself or by new stings), those allergic to bee venom, and those who suffer from systemic mastocytosis. In those cases, it is recommended to continue with the administration of specific immunotherapy for over 5 years.

Reactions caused by venom immunotherapy occur in $2-20 \%$ of the patients who receive it. Said reactions are more common in patients who are allergic to bee venom, they occur with greater frequency during the increasing stage, and when ultra-rush immunotherapy is used. Patients who receive beta-blockers may suffer more severe reactions ${ }^{1,6}$.

Premedications with antihistamines can increase the safety of venom immunotherapy, along with the use of omalizumab in those cases of coexisting systemic mastocytosis, or in those where severe systemic reactions have occurred during immunotherapy. It seems to be a more efficient measure to reduce the risk of new systemic reactions, even though observed results have varied $^{1,6}$.

Patients who have suffered anaphylaxis caused by Hymenoptera stings ought to be educated on preventative measures, as well as trained for the autoadministration of epinephrine in the case of future similar events. In addition, the use of a medical identification should be recommended, one which alerts caregivers to the diagnosis of allergy to Hymenoptera stings.

\section{Ethical disclosures}

Protection of human and animal subjects. The authors declare that no experiments were performed on humans or animals for this study.

Confidentiality of data. The authors declare that no patient data appear in this article.

Right to privacy and informed consent. The authors declare that no patient data appear in this article.

\section{References}

1. Golden DB, Moffitt J, Nicklas RA, et al. Stinging insect hypersensitivity: a practice parameter update 2011. J Allergy Clin Immunol. 2011;127:852-4.

2. Severino $M$, Bonadonna $P$, Passalacqua $G$. Large local reactions from stinging insects: from epidemiology to management. Curr Opin Allergy Clin Immunol. 2009:9:334-7.

3. Demain JG, Minaei AA, Tracy JM. Anaphylaxis and insect allergy. Curr Opin Allergy Clin Immunol. 2010;10:318-22.

4. Bilò MB. Anaphylaxis caused by Hymenoptera stings: from epidemiology to treatment. Allergy. 2011;66 Suppl 95:35-7.

5. Bilò MB, Bonifazi F. The natural history and epidemiology of insect venom allergy: clinical implications. Clin Exp Allergy. 2009:39:1467-76.

6. Golden DBK, Demain J, Freeman T, Graft D, et al. Stinging insect hypersensitivity. A practice parameter update 2016. Ann Allergy Asthma Immunol. 2017; 118:28-54. 